\title{
BDII, Rat Strain
}

National Cancer Institute

\section{Source}

National Cancer Institute. BDII, Rat Strain. NCI Thesaurus. Code C14388.

Frequent microphthalmia. Genetic markers described for sublines of this strain in the USA differ from those in Europe, suggesting that the US sublines were contaminated at some time in the past with LEW (Czerwinski and Crowell 1986). Mean life-span of BDII/Han 33.6 +/-4.7 months in males and 22.5+/-4.4 months in females, with $91 \%$ of females developing endometrial carcinomas, but with a more varied range of tumors in males. These include 26\% adrenal pheochromocytomas, 23\% histiocytic sarcomas, 18\% pituitary tumors and $14 \%$ hemangiomas. About $12 \%$ of aged male BDII/Han develop diverticula of the stomach (Kaspareit-Rittinghausen and Deerberg 1987). High incidence of hormone-dependent endometrial adenocarcinoma. 